\title{
Automated Induction with Constrained Tree Automata ${ }^{\star}$
}

\author{
Adel Bouhoula ${ }^{1}$ and Florent Jacquemard ${ }^{2}$ \\ ${ }^{1}$ Higher School of Communications of Tunis (Sup'Com), \\ University of November 7th at Carthage, Tunisia \\ adel.bouhoula@supcom.rnu.tn \\ ${ }^{2}$ INRIA and LSV, CNRS/ENS Cachan, France \\ florent.jacquemard@inria.fr
}

\begin{abstract}
We propose a procedure for automated implicit inductive theorem proving for equational specifications made of rewrite rules with conditions and constraints. The constraints are interpreted over constructor terms (representing data values), and may express syntactic equality, disequality, ordering and also membership in a fixed tree language. Constrained equational axioms between constructor terms are supported and can be used in order to specify complex data structures like sets, sorted lists, trees, powerlists...

Our procedure is based on tree grammars with constraints, a formalism which can describe exactly the initial model of the given specification (when it is sufficiently complete and terminating). They are used in the inductive proofs first as an induction scheme for the generation of subgoals at induction steps, second for checking validity and redundancy criteria by reduction to an emptiness problem, and third for defining and solving membership constraints.

We show that the procedure is sound and refutationally complete. It generalizes former test set induction techniques and yields natural proofs for several non-trivial examples presented in the paper, these examples are difficult (if not impossible) to specify and carry on automatically with other induction procedures.
\end{abstract}

\section{Introduction}

Given a specification $\mathcal{R}$ of a program or system $S$ made of equational Horn clauses, proving a property $P$ for $S$ generally amounts to show the validity of $P$ in the minimal Herbrand model of $\mathcal{R}$, also called initial model of $\mathcal{R}$ (inductive validity). In this perspective, it is important to have automated induction theorem proving procedures supporting a specification language expressive enough to axiomatize complex data structures like sets, sorted lists, powerlists, complete binary trees, etc. Moreover, it is also important to be able to automatically generate induction schemas used for inductive proofs in order to minimize user interaction. However,

\footnotetext{
* A long version of this extended abstract is available as a research report 3 .

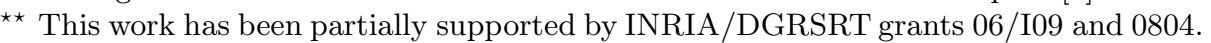


theories of complex data structures generate complex induction schemes, and the automation of inductive proofs is therefore difficult for such theories.

It is common to assume that $\mathcal{R}$ is built with constructor function symbols (to construct terms representing data) and defined symbols (representing the operations defined on constructor terms). Assuming in addition the sufficient completeness of $\mathcal{R}$ (every ground (variable-free) term is reducible, using the axioms of $\mathcal{R}$, to a constructor term) and the strong termination of the axioms of $\mathcal{R}$, a set of representants for the initial model of $\mathcal{R}$ (the model in which we want to proof the validity of conjectures) is the set of ground constructor terms not reducible by $\mathcal{R}_{\mathcal{C}}$ (the subset of equations of $\mathcal{R}$ between terms made of constructor symbols), called constructor normal forms.

In the case where the constructors are free $\left(\mathcal{R}_{\mathcal{C}}=\emptyset\right)$, the set of constructor normal forms is simply the set of ground terms built with constructors and it is very easy to define an induction schema. This situation is therefore convenient for inductive reasoning, and many inductive theorem provers require free constructors, termination and sufficient completeness. However, it is not expressive enough to define complex data structures. With rewrite rules between constructors, the definition of induction schema is more complex, and requires a finite description of the set of constructor normal-forms. Some progress has been done e.g. in [4] and [5] in the direction of handling specification with non-free constructors, with severe restrictions (see related work below).

Tree automata (TA), or equivalently regular tree grammars, permit a finite representation of the set of constructor normal-forms when $\mathcal{R}_{\mathcal{C}}$ is a left-linear rewrite system (set of rewrite rules without multiple occurrences of variables in their left-hand-sides). Indeed, on one hand TA can do linear pattern-matching, hence they can recognize terms which are reducible by $\mathcal{R}_{\mathcal{C}}$, and on the other hand, the class of TA languages is closed under complementation. When the axioms of $\mathcal{R}_{\mathcal{C}}$ are not linear, or are constrained, some extensions of TA (or grammars) are necessary, with transitions able to check constraints on the term in input, see e.g. 8].

In this paper, we propose a framework for inductive theorem proving for theories containing constrained rewrite rules between constructor terms and conditional and constrained rewrite rules for defined functions. The key idea is a strong and natural integration of tree grammars with constraints in an implicit induction procedure, where they are used as induction schema. Very roughly, our procedure starts with the automatic computation of an induction schema, in the form of a constrained tree grammar generating constructor normal form. This grammar is used later for the generation of subgoals from a conjecture $C$, by instantiation of variables using the grammar's production rules, triggering induction steps during the proof. All generated subgoals are either deleted, following some criteria, or they are reduced, using axioms or induction hypotheses, or conjectures not yet proved, providing that they are smaller than the goal to be proved. Reduced subgoals become then new conjectures and $C$ becomes an induction hypothesis. Moreover, constrained tree grammars are used as a decision procedure for checking the deletion criteria during induction steps. 
Our method subsumes former test set induction procedures like [6]14], by reusing former theoretical works on tree automata with constraints. It is sound and refutationally complete (any conjecture that is not valid in the initial model will be disproved) when $\mathcal{R}$ is sufficiently complete and the constructor subsystem $\mathcal{R}_{\mathcal{C}}$ is terminating. Without the above hypotheses, it still remains sound and refutationally complete for a restricted kind of conjectures, where all the variables are constrained to belong to the language of constructor normal forms. This restriction is expressible in the specification language (see below). When the procedure fails, it implies that the conjecture is not an inductive theorem, provided that $\mathcal{R}$ is strongly complete (a stronger condition for sufficient completeness) and ground confluent. There is no requirement for termination of the whole set of rules $\mathcal{R}$, unlike [61], but instead only for separate termination of the respective sets of rules for defined function and for the constructors.

Moreover, if a conjecture $C$ restricted as above is proved in a sufficiently complete specification $\mathcal{R}$ and $\mathcal{R}$ is further consistently extended into $\mathcal{R}^{\prime}$ with additional axioms for specifying partial (non-constructor) functions, then the former proof of $C$ remains valid in $\mathcal{R}^{\prime}$, see Section 5 .

The support of constraints permits in some cases to use the constrained completion technique of [16] in order to transform a non-terminating theory into a terminating one, by the addition of ordering constraints in constructor rules, see Section 4.5. It permits in particular to make proofs modulo non orientable axioms, without having to modify the core of our procedure.

We shall consider a specification of ordered lists as a running example throughout the paper. Consider first non-stuttering lists (lists which do not contain two equal successive elements) built with the constructor symbols $\emptyset$ (empty list) and ins (list insertion) and following this rewrite rule:

$$
\operatorname{ins}(x, \operatorname{ins}(x, y)) \rightarrow \operatorname{ins}(x, y)
$$

Rewrite rules can be enriched with constraints built on predicates with a fixed interpretation on ground constructor terms. For example, using ordering constraints built with $\succ$ we can specify ordered lists by the following axiom:

$$
\operatorname{ins}\left(x_{1}, \operatorname{ins}\left(x_{2}, y\right)\right) \rightarrow \operatorname{ins}\left(x_{2}, \operatorname{ins}\left(x_{1}, y\right)\right) \llbracket x_{1} \succ x_{2} \rrbracket
$$

Another interesting example is the case of membership constraints of the form $x: L$ where $L$ is a fixed regular tree language (containing only terms made of constructor symbols). We consider also stronger constraints which restrict constructor terms to be in normal form (i.e. not reducible by the axioms). Let us come back to the example of non-stuttering sorted lists (sorted lists without duplication), and add to the above rules the axioms below which define a membership predicate $\Subset$, using the information that lists are sorted:

$$
\begin{aligned}
x \Subset \emptyset & \rightarrow \text { false } & & \left(\mathrm{m}_{0}^{\prime}\right) \\
x_{1} \Subset \text { ins }\left(x_{2}, y_{2}\right) & \rightarrow \text { true } \llbracket x_{1} \approx x_{2} \rrbracket & & \left(\mathrm{m}_{1}^{\prime}\right) \\
x_{1} \Subset y_{1} & \rightarrow \text { false } \llbracket y_{1} \approx \text { ins }\left(x_{2}, y_{2}\right), x_{1} \prec x_{2}, y_{1}: \mathrm{NF} \rrbracket & & \left(\mathrm{m}_{2}^{\prime}\right) \\
x_{1} \Subset \text { ins }\left(x_{2}, y_{2}\right) & \rightarrow x_{1} \Subset y_{2} \llbracket x_{2} \prec x_{1} \rrbracket & & \left(\mathrm{m}_{3}^{\prime}\right)
\end{aligned}
$$


The constraint $y_{1}$ :NF expresses the fact that this subterm is a constructor term in normal form, i.e. that it is a sorted list. Without this constraint, the specification would be inconsistent. Indeed, let us consider the ground term $t=$ $0 \Subset \operatorname{ins}(s(0)$, ins $(0, \emptyset))$. This term $t$ can be reduced into both true and false, since ins $(s(0)$, ins $(0, \emptyset))$ is not in normal form. Using constraints of the form . : NF as above also permits the user to specify, directly in the rewrite rules, some adhoc reduction strategies for the application of rewriting. Such strategies include for instance several refinements of the innermost strategy which corresponds to the call by value computation in functional programming languages, where arguments are fully evaluated before the function application.

Related work. The principle of our procedure is close to test-set induction approaches [61]. The real novelty here is that test-sets are replaced by constrained tree grammars, the latter being more precise induction schemes. Indeed, they provide an exact finite description of the initial model of the given specification, (under some assumptions like sufficient completeness and termination for axioms), whereas cover-sets and test-sets are over-approximative in similar cases.

The first author and Jouannaud [4 have used tree automata techniques to generalize test set induction to specifications with non-free constructors. This work has been generalized in 5 for membership equational logic. These approaches, unlike the procedure presented in this paper, work by transforming the initial specification in order to get rid of rewrite rules for constructors. Moreover, the axioms for constructors are assumed to be unconstrained and unconditional leftlinear rewrite rules, which is still too restrictive for the specification of structures like sets or sorted lists...

Kapur [15] has proposed a method (implemented in the system RRL) for mechanizing cover set induction if the constructors are not free. This handles in particular the specification of powerlists or sorted lists. We show in Section [5] how our method can address similar problems.

We describe in 3 two proofs, done resp. by Jared Davis and Sorin Stratulat, of a conjecture on sorted lists, done resp. by Jared Davis and Sorin Stratulat, with ACL2 using a library for ordered sets [12] and with SPIKE [6|1|17]. Both proofs require the addition of non-trivial lemmas whereas our procedure can prove the conjecture without additional lemma.

\section{Preliminaries}

The reader is assumed familiar with the basic notions of term rewriting [13] and first-order logic. Notions and notations not defined here are standard.

Terms and substitutions. We assume given a many sorted signature $(\mathcal{S}, \mathcal{F})$ (or simply $\mathcal{F}$, for short) where $\mathcal{S}$ is a set of sorts and $\mathcal{F}$ is a finite set of function symbols with arities. We assume moreover that the signature $\mathcal{F}$ comes in two parts, $\mathcal{F}=\mathcal{C} \uplus \mathcal{D}$ where $\mathcal{C}$ a set of constructor symbols, and $\mathcal{D}$ is a set of defined symbols. Let $\mathcal{X}$ be a family of sorted variables. We sometimes denote variables with sort exponent like $x^{S}$ in order to indicate that $x$ has sort $S \in \mathcal{S}$. The set 
of well-sorted terms over $\mathcal{F}$ (resp. constructor well-sorted terms) with variables in $\mathcal{X}$ will be denoted by $\mathcal{T}(\mathcal{F}, \mathcal{X})(\operatorname{resp} . \mathcal{T}(\mathcal{C}, \mathcal{X})$ ). The subset of $\mathcal{T}(\mathcal{F}, \mathcal{X})$ (resp. $\mathcal{T}(\mathcal{C}, \mathcal{X})$ ) of variable-free terms, or ground terms, is denoted $\mathcal{T}(\mathcal{F})($ resp. $\mathcal{T}(\mathcal{C})$ ). We assume that each sort contains a ground term. The sort of a term $t \in \mathcal{T}(\mathcal{F}, \mathcal{X})$ is denoted $\operatorname{sort}(t)$.

A term $t$ is identified as usual with a function from its set of positions (strings of positive integers) $\mathcal{P} o s(t)$ to symbols of $\mathcal{F}$ and $\mathcal{X}$, where positions are strings of positive integers. We denote the empty string (root position) by $\Lambda$. The length of a position $p$ is denoted $|p|$. The depth of a term $t$, denoted $d(t)$, is the maximum of $\left\{|p| \mid p \in \mathcal{P}_{o s}(t)\right\}$. The subterm of $t$ at position $p$ is denoted by $\left.t\right|_{p}$. The result of replacing $\left.t\right|_{p}$ with $s$ at position $p$ in $t$ is denoted by $t[s]_{p}$. This notation is also used to indicate that $s$ is a subterm of $t$, in which case $p$ may be omitted. We denote the set of variables occurring in $t$ by $\operatorname{var}(t)$. A term $t$ is linear if every variable of $\operatorname{var}(t)$ occurs exactly once in $t$.

A substitution is a finite mapping $\left\{x_{1} \mapsto t_{1}, \ldots, x_{n} \mapsto t_{n}\right\}$ where $x_{1}, \ldots, x_{n} \in$ $\mathcal{X}$ and $t_{1}, \ldots t_{n} \in \mathcal{T}(\mathcal{F}, \mathcal{X})$. As usual, we identify substitutions with their morphism extension to terms. A variable renaming is a substitution mapping variables to variables. We use postfix notation for substitutions application and composition. A substitution $\sigma$ is grounding for a term $t$ if $t \sigma$ is ground.

Constraints and constrained terms. We assume given a constraint language $\mathcal{L}$, which is a finite set of predicate symbols with a recursive Boolean interpretation in the domain of ground constructor terms of $\mathcal{T}(\mathcal{C})$. Typically, $\mathcal{L}$ may contain the syntactic equality $\approx$. (syntactic disequality . $\not$.), some (recursive) simplification ordering . $\prec$. on ground constructor terms (for instance a lexicographic path ordering $[13$ ), and membership . :L to a fixed tree language $L \subseteq \mathcal{T}(\mathcal{C})$ (like for instance the languages of well sorted terms or constructor terms in normal-form). Constraints on the language $\mathcal{L}$ are Boolean combinations of atoms of the form $P\left(t_{1}, \ldots, t_{n}\right)$ where $P \in \mathcal{L}$ and $t_{1}, \ldots, t_{n} \in \mathcal{T}(\mathcal{C}, \mathcal{X})$. By convention, an empty combination is interpreted to true.

The application of substitutions is extended from terms to constraints in a straightforward way, and we may therefore define a solution for a constraint $c$ as a (constructor) substitution $\sigma$ grounding for all terms in $c$ and such that $c \sigma$ is interpreted to true. The set of solutions of the constraint $c$ is denoted $\operatorname{sol}(c)$. A constraint $c$ is satisfiable if $\operatorname{sol}(c) \neq \emptyset$ (and unsatisfiable otherwise).

A constrained term $t \llbracket c \rrbracket$ is a linear term $t \in \mathcal{T}(\mathcal{F}, \mathcal{X})$ together with a constraint $c$, which may share some variables with $t$. Note that the assumption that $t$ is linear is not restrictive, since any non linearity may be expressed in the constraint, for instance $f(x, x) \llbracket c \rrbracket$ is semantically equivalent to $f\left(x, x^{\prime}\right) \llbracket c \wedge x \approx x^{\prime} \rrbracket$, where the variable $x^{\prime}$ does not occur in $c$.

Constrained clauses. A literal is an equation $s=t$ or a disequation $s \neq t$ or an oriented equation $s \rightarrow t$ between two terms. A constrained clause $C \llbracket c \rrbracket$ is a disjunction $C$ of literals together with a constraint $c$. A constrained clause $C \llbracket c \rrbracket$ is said to subsume a constrained clause $C^{\prime} \llbracket c^{\prime} \rrbracket$ if there is a substitution $\sigma$ such that $C \sigma$ is a sub-clause of $C^{\prime}$ and $c^{\prime} \wedge \neg c \sigma$ is unsatisfiable. 
A tautology is a constrained clause $s_{1}=t_{1} \vee \ldots \vee s_{n}=t_{n} \llbracket d \rrbracket$ such that $d$ is a conjunction of equational constraints, $d=u_{1} \approx v_{1} \wedge \ldots \wedge u_{k} \approx v_{k}$ and there exists $i \in[1 . . n]$ such that $s_{i} \sigma=t_{i} \sigma$ where $\sigma$ is the mgu of $d$.

Constrained rewriting. A conditional constrained rewrite rule is a constrained clause of the form $\Gamma \Rightarrow l \rightarrow r \llbracket c \rrbracket$ such that $\Gamma$ is a conjunction of equations, called the condition of the rule, the terms $l$ and $r$ (called resp. left- and right-hand side) are linear and have the same sort, and $c$ is a constraint. When the condition $\Gamma$ is empty, it is called a constrained rewrite rule. A set of conditional constrained, resp. constrained, rules is called a conditional constrained (resp. constrained) rewrite system. Let $\mathcal{R}$ be a conditional constrained rewrite system. The relation $s \llbracket d \rrbracket$ rewrites to $t \llbracket d \rrbracket$ by $\mathcal{R}$, denoted $s \llbracket d \rrbracket \overrightarrow{\mathcal{R}} t \llbracket d \rrbracket$, is defined recursively by the existence of a rule $\rho \equiv \Gamma \Rightarrow \ell \rightarrow r \llbracket c \rrbracket \in \mathcal{R}$, a position $p \in \mathcal{P} o s(s)$, and a substitution $\sigma$ such that $\left.s\right|_{p}=\ell \sigma,\left.t\right|_{p}=r \sigma, d \sigma \wedge \neg c \sigma$ is unsatisfiable, and $u \sigma \downarrow_{\mathcal{R}} v \sigma$ for all $u=v \in \Gamma$. The transitive and reflexive transitive closures, of $\overrightarrow{\mathcal{R}}$ are denoted $\stackrel{+}{\mathcal{R}}$ and $\stackrel{*}{\mathcal{R}}$, and $u \downarrow_{\mathcal{R}} v$ stands for $\exists w, u \stackrel{*}{\mathcal{R}} w \stackrel{*}{\mathcal{R}} v$.

Note the semantical difference between conditions and constraints in rewrite rules. The validity of the condition is defined wrt the system $\mathcal{R}$ whereas the interpretation of constraint is fixed and independent from $\mathcal{R}$.

A constrained term $s \llbracket c \rrbracket$ is reducible by $\mathcal{R}$ if there is some $t \llbracket c \rrbracket$ such that $s \llbracket c \rrbracket \overrightarrow{\mathcal{R}} t \llbracket c \rrbracket$. Otherwise $s \llbracket c \rrbracket$ is called irreducible, or an $\mathcal{R}$-normal form. A substitution $\sigma$ is irreducible by $\mathcal{R}$ if its image contains only $\mathcal{R}$-normal forms. A constrained term $t \llbracket c \rrbracket$ is ground reducible (resp. ground irreducible) if $t \sigma$ is reducible (resp. irreducible) for every irreducible solution $\sigma$ of $c$ grounding for $t$.

The system $\mathcal{R}$ is terminating if there is no infinite sequence $t_{1} \underset{\mathcal{R}}{\longrightarrow} t_{2} \overrightarrow{\mathcal{R}_{*}} \ldots$,

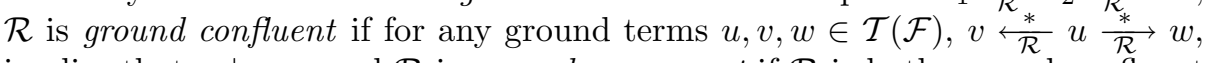
implies that $v \downarrow_{\mathcal{R}} w$, and $\mathcal{R}$ is ground convergent if $\mathcal{R}$ is both ground confluent and terminating. The depth of a non-empty set $\mathcal{R}$ of rules, denoted $d(\mathcal{R})$, is the maximum of the depths of the left-hand sides of rules in $\mathcal{R}$.

Constructor specifications. We assume from now on given a conditional constrained rewrite system $\mathcal{R}$. The subset of $\mathcal{R}$ containing only function symbols from $\mathcal{C}$ is denoted $\mathcal{R}_{\mathcal{C}}$ and $\mathcal{R} \backslash \mathcal{R}_{\mathcal{C}}$ is denoted $\mathcal{R}_{\mathcal{D}}$.

Inductive theorems. A clause $C$ is a deductive theorem of $\mathcal{R}$ (denoted $\mathcal{R} \models C$ ) if it is valid in any model of $\mathcal{R}$. A clause $C$ is an inductive theorem of $\mathcal{R}$ (denoted $\mathcal{R} \models_{\mathcal{I} n d} C$ ) iff for all for all substitution $\sigma$ grounding for $C, \mathcal{R} \models C \sigma$.

We shall need below to generalize the definition of inductive theorems to constrained clauses as follows: a constrained clause $C \llbracket c \rrbracket$ is an inductive theorem of $\mathcal{R}$ (denoted $\mathcal{R} \models_{\mathcal{I} n d} C \llbracket c \rrbracket$ ) if for all substitutions $\sigma \in \operatorname{sol}(c)$ grounding for $C$ we have $\mathcal{R} \models C \sigma$.

Completeness. A function symbol $f \in \mathcal{D}$ is sufficiently complete wrt $\mathcal{R}$ iff for all $t_{1}, \ldots, t_{n} \in \mathcal{T}(\mathcal{C})$, there exists $t$ in $\mathcal{T}(\mathcal{C})$ such that $f\left(t_{1}, \ldots, t_{n}\right) \stackrel{+}{\mathcal{R}} t$. We say that the system $\mathcal{R}$ is sufficiently complete iff every defined operator $f \in \mathcal{D}$ is sufficiently complete wrt $\mathcal{R}$. Let $f \in \mathcal{D}$ be a function symbol and let:

$$
\left\{\Gamma_{1} \Rightarrow f\left(t_{1}^{1}, \ldots, t_{k}^{1}\right) \rightarrow r_{1} \llbracket c_{1} \rrbracket, \ldots, \Gamma_{n} \Rightarrow f\left(t_{1}^{n}, \ldots, t_{k}^{n}\right) \rightarrow r_{n} \llbracket c_{n} \rrbracket\right\}
$$


be a maximal subset of rules of $\mathcal{R}_{\mathcal{D}}$ whose left-hand sides are identical up to variable renamings $\mu_{1}, \ldots, \mu_{n}$, i.e. $f\left(t_{1}^{1}, \ldots, t_{k}^{1}\right) \mu_{1}=f\left(t_{1}^{2}, \ldots, t_{k}^{2}\right) \mu_{2}=$ $\ldots f\left(t_{1}^{n}, \ldots, t_{k}^{n}\right) \mu_{n}$. We say that $f$ is strongly complete wrt $\mathcal{R}$ (see [1]) if $f$ is sufficiently complete wrt $\mathcal{R}$ and $\mathcal{R} \models_{\mathcal{I} n d} \Gamma_{1} \mu_{1} \llbracket c_{1} \mu_{1} \rrbracket \vee \ldots \vee \Gamma_{n} \mu_{n} \llbracket c_{n} \mu_{n} \rrbracket$ for every subset of $\mathcal{R}$ as above. The system $\mathcal{R}$ is said strongly complete if every function symbol $f \in \mathcal{D}$ is strongly complete wrt $\mathcal{R}$.

\section{Constrained Grammars}

Constrained tree grammars have been introduced in (7), in the context of automated induction. The idea of using such formalism for induction theorem proving is also in e.g. 4 410, because it is known that they can generate the languages of normal-forms for arbitrary term rewriting systems.

We present in this section the definitions and results suited to our purpose.

Definition 1. A constrained grammar $\mathcal{G}=(Q, \Delta)$ is given by: 1. a finite set $Q$ of non-terminals of the form $\left\llcorner_{\lrcorner} u_{\lrcorner}\right.$, where $u$ is a linear term of $\mathcal{T}(\mathcal{F}, \mathcal{X})$, 2. a finite set $\Delta$ of production rules of the form $\left\llcorner v_{\lrcorner}:=f\left(\left\llcorner u_{1}, \ldots,\left\llcorner u_{n\lrcorner}\right) \llbracket c \rrbracket\right.\right.\right.$ where $f \in \mathcal{F}$, $\left\llcorner v_{\lrcorner},\left\llcorner u_{1}, \ldots,\left\llcorner u_{n} \in Q\right.\right.\right.$ (modulo variable renaming) and $c$ is a constraint.

The non-terminals are always considered modulo variable renaming. In particular, we assume $w \log$ (for technical convenience) that the above term $f\left(u_{1}, \ldots, u_{n}\right)$ is linear and that $\operatorname{var}(v) \cap \operatorname{var}\left(f\left(u_{1}, \ldots, u_{n}\right)\right)=\emptyset$.

\subsection{Term Generation}

We associate to a given constrained grammar $\mathcal{G}=(Q, \Delta)$ a finite set of new unary predicates of constraint of the form.$:\left\llcorner u_{\lrcorner}\right.$, where $\left\llcorner u_{\lrcorner} \in Q\right.$ (modulo variable renaming). Constraints of the form $t:\llcorner u\lrcorner$ called membership constraints and their interpretation is given below. The production relation between constrained terms $\vdash_{\mathcal{G}}^{y}$ is defined by:

$$
t[y] \llbracket y:\left\llcorner v_{\lrcorner} \wedge d \rrbracket \vdash_{\mathcal{G}}^{y} t\left[f\left(y_{1}, \ldots, y_{n}\right)\right] \llbracket y_{1}:\left\llcorner u_{1}\right\lrcorner \wedge \ldots \wedge y_{n}:\left\llcorner u_{n}\right\lrcorner \wedge \wedge d \tau \rrbracket\right.
$$

if there exists $\left\llcorner v_{\lrcorner}:=f\left(\left\llcorner u_{1}, \ldots,\left\llcorner u_{n}\right\lrcorner\right) \llbracket c \rrbracket \in \Delta\right.\right.$ such that $f\left(u_{1}, \ldots, u_{n}\right)=$ $v \tau$, and $y_{1}, \ldots, y_{n}$ are fresh variables. The variable $y$, constrained to be in the language defined by the non-terminal $\left\llcorner v_{\lrcorner}\right.$is replaced by $f\left(y_{1}, \ldots, y_{n}\right)$ where the variables $y_{1}, \ldots, y_{n}$ are constrained to the respective languages of non-terminals $\left\llcorner u_{1}\right\lrcorner, \ldots,\left\llcorner u_{n}\right.$. The union of the relations $\vdash_{\mathcal{G}}^{y}$ for all $y$ is denoted $\vdash_{\mathcal{G}}$ and the reflexive transitive and transitive closures of the relation $\vdash_{\mathcal{G}}$ are respectively denoted by $\vdash_{\mathcal{G}}^{*}$ and $\vdash_{\mathcal{G}}^{+}$( $\mathcal{G}$ may be omitted).

Definition 2. The language $L\left(\mathcal{G},\left\llcorner u_{\lrcorner}\right)\right.$is the set of ground terms $t$ generated by $\mathcal{G}$ from a non-terminal $\left\llcorner u_{\lrcorner}\right.$, i.e. such that $y \llbracket y:\llcorner u\lrcorner \rrbracket \vdash^{*} t \llbracket c \rrbracket$ where $c$ is satisfiable.

Given $Q^{\prime} \subseteq Q$, we write $L\left(\mathcal{G}, Q^{\prime}\right)=\bigcup u_{\lrcorner} \in Q^{\prime} L\left(\mathcal{G},\left\llcorner u_{\lrcorner}\right)\right.$and $L(\mathcal{G})=L(\mathcal{G}, Q)$. Given a constrained grammar $\mathcal{G}=(Q, \Delta)$, we can now define $\operatorname{sol}\left(t:\left\llcorner u_{\lrcorner}\right)\right.$, where $\left\llcorner u_{\lrcorner} \in Q\right.$, as $\left\{\sigma \mid t \sigma \in L\left(\mathcal{G},\left\llcorner u_{\lrcorner}\right)\right\}\right.$. 
Example 1. Let us consider the sort Nat of natural integers built with the constructor symbols 0 and $s$. These terms are generated by the grammar with production rules $\left\llcorner x_{\lrcorner}^{\mathrm{Nat}}:=0\right.$ and $\left\llcorner x_{\lrcorner}^{\mathrm{Nat}}:=s\left({ }_{\llcorner} x_{2}\right\lrcorner{ }^{\mathrm{Nat}}\right)$.

\subsection{Normal Forms}

In 3], we present the automatic construction of a constrained grammar $\mathcal{G}_{\mathrm{NF}}\left(\mathcal{R}_{\mathcal{C}}\right)=\left(Q_{\mathrm{NF}}\left(\mathcal{R}_{\mathcal{C}}\right), \Delta_{\mathrm{NF}}\left(\mathcal{R}_{\mathcal{C}}\right)\right)$ which generates the language of ground $\mathcal{R}_{\mathcal{C}^{-}}$ normal forms. Its construction is a generalization of the one of 9]. Intuitively, it corresponds to the complementation and completion of a grammar for $\mathcal{R}_{\mathcal{C}^{-}}$ reducible terms (such a grammar does mainly pattern matching of left members of rewrite rules), where every subset of states (for the complementation) is represented by the most general common instance of its elements (if they are unifiable). Due to space limitations, we cannot describe the general construction of $\mathcal{G}_{\mathrm{NF}}\left(\mathcal{R}_{\mathcal{C}}\right)$ here but we rather present the case where $\mathcal{R}_{\mathcal{C}}$ contains the constructor axioms given in introduction.

Example 2. Let $\mathcal{R}_{\mathcal{C}}$ contain the axioms $\left(\mathbb{C _ { 0 }}\right)$ and $\left(\mathbb{C}_{1}\right)$ given in introduction. Let $\mathcal{G}_{\mathrm{NF}}\left(\mathcal{R}_{\mathcal{C}}\right)$ contain the two productions rules given Example 1 and $x_{\lrcorner}^{\mathrm{Set}}:=$ $\emptyset, \quad$ ins $(x, y)\lrcorner:=\operatorname{ins}\left(\left\llcorner x_{\lrcorner}^{\mathrm{Nat}},{ }_{\llcorner} x_{\lrcorner}^{\mathrm{Set}}\right)\right.$ (for singleton lists) and ${ }_{\llcorner}$ins $\left.(x, y)\right\lrcorner:=$ ins $\left(\left\llcorner x_{\lrcorner}^{\mathrm{Nat}}, \iota_{\llcorner}\right.\right.$ins $\left.\left.\left(x_{2}, y_{2}\right)\right\lrcorner\right) \llbracket x^{\text {Nat }} \prec x_{2} \rrbracket$. Note that the variables in the non terminal ins $\left(x_{2}, y_{2}\right)$, in the right member of the latter production rule have been renamed in order to be distinguished from the variables in the non terminal in the left member. This grammar $\mathcal{G}_{\mathrm{NF}}\left(\mathcal{R}_{\mathcal{C}}\right)$ generates the set of ground constructor terms in normal-form for $\mathcal{R}_{\mathcal{C}}$. They represent the ordered lists of natural numbers (of sort List).

\section{Inference System}

In this section, we present an inference system for our inductive theorem proving procedure. The principle is the following: given a goal (conjecture) $C$, we use the grammar $\mathcal{G}_{\mathrm{NF}}\left(\mathcal{R}_{\mathcal{C}}\right)$ of Section 3.2 in order to expand $C$ into some subgoals. All the generated subgoals must then either be deleted, following some criteria, or be reduced, using axioms or induction hypotheses, or conjectures not yet proved, providing that they are smaller than the goal to be proved. Reduced subgoals become then new conjectures and $C$ becomes an induction hypothesis.

The deletion criteria include tautologies, forward subsumption, clauses with an unsatisfiable constraint, and constructor clause that can be detected as inductively valid, under some conditions defined precisely below. The decision of these criteria, using $\mathcal{G}_{\mathrm{NF}}\left(\mathcal{R}_{\mathcal{C}}\right)$, is discussed in Section 4.6 .

The reduction of subgoals is performed with the rules defined in Sections 4.1 and 4.2. If a subgoal generated cannot be deleted or reduced, then the procedure stop with a refutation (the initial goal is not an inductive theorem of $\mathcal{R}$ ). If every subgoal is deleted, then the initial goal is an inductive theorem of $\mathcal{R}$.

The procedure may not terminate (the conditions in inference rules other than the deletion criteria are recursive calls of the procedure of the form $\mathcal{R} \models_{\text {Ind }}$ 
subgoal). In this case appropriate lemmas should be added by the user in order to achieve termination.

\subsection{Simplification Rules for Defined Functions}

Our procedure uses the simplification rules for defined symbols presented in Figure 1. They simplify constrained clauses according to $\mathcal{R}_{\mathcal{D}}$ and to a set $\mathcal{H}$ of induction hypotheses (constrained clauses), which is given as the second component of the left-hand sides of rules. Inductive Rewriting simplifies goals using the axioms as well as instances of the induction hypotheses of $\mathcal{H}$, provided that they are smaller than the goal. The underlying induction principle is based on a well-founded ordering $\gg$ on constrained clauses (see [3]). This approach is more general than structural induction which is more restrictive concerning simplification with induction hypotheses (see e.g. 6]). Inductive Contextual Rewriting can be viewed as a generalization of a rule in 18 to handle constraints by recursively discharging them as inductive conjectures. Rewrite Splitting simplifies a clause which contains a subterm matching some left member of rule of $\mathcal{R}_{\mathcal{D}}$. This inference checks moreover that all cases are covered for the application of $\mathcal{R}_{\mathcal{D}}$, i.e. that for each ground substitution $\tau$, the conditions and the constraints of at least one rule is true wrt $\tau$. Note that this condition is always true when $\mathcal{R}$ is sufficiently complete, and hence that this check is superfluous in this case. Inductive Deletion deletes tautologies and clauses with unsatisfiable constraints.

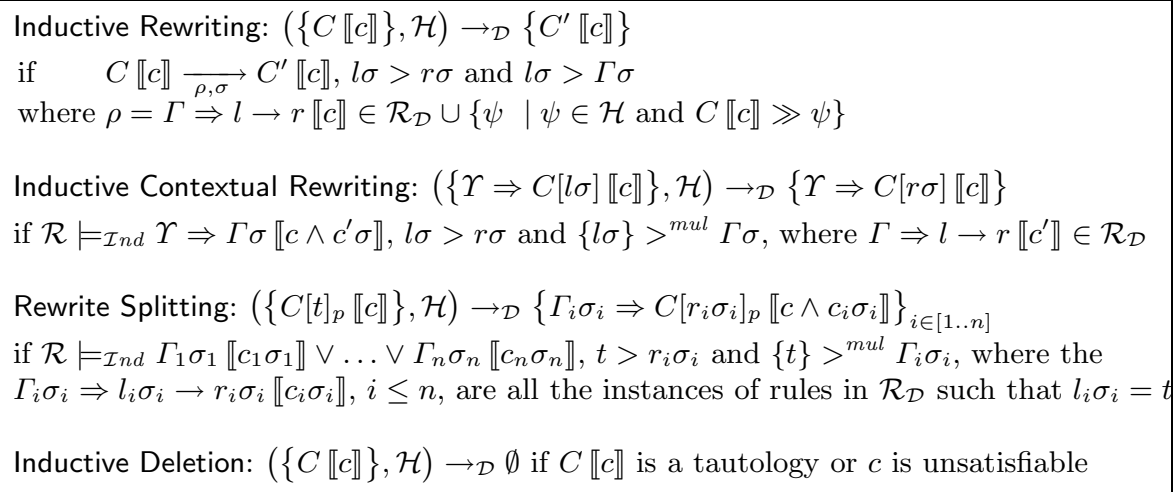

Fig. 1. Simplification Rules for Defined Functions

\subsection{Simplification Rules for Constructors}

The simplification rules for constructors are presented in Figure2. Rewriting simplifies goals with axioms from $\mathcal{R}_{\mathcal{C}}$. Partial Splitting eliminates ground reducible terms in a constrained clause $C \llbracket c \rrbracket$ by adding to $C \llbracket c \rrbracket$ the negation of constraint of some rules of $\mathcal{R}_{\mathcal{C}}$. Therefore, the saturated application of Partial splitting and Rewriting will always lead to Deletion or to ground irreducible constructor clauses. Finally, Deletion and Validity remove respectively tautologies and clauses with unsatisfiable constraints, and ground irreducible constructor theorems of $\mathcal{R}$. 


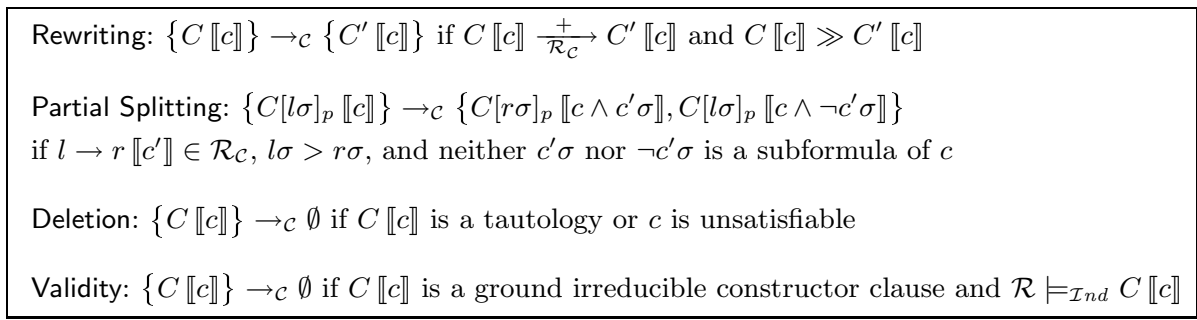

Fig. 2. Simplification Rules for Constructors

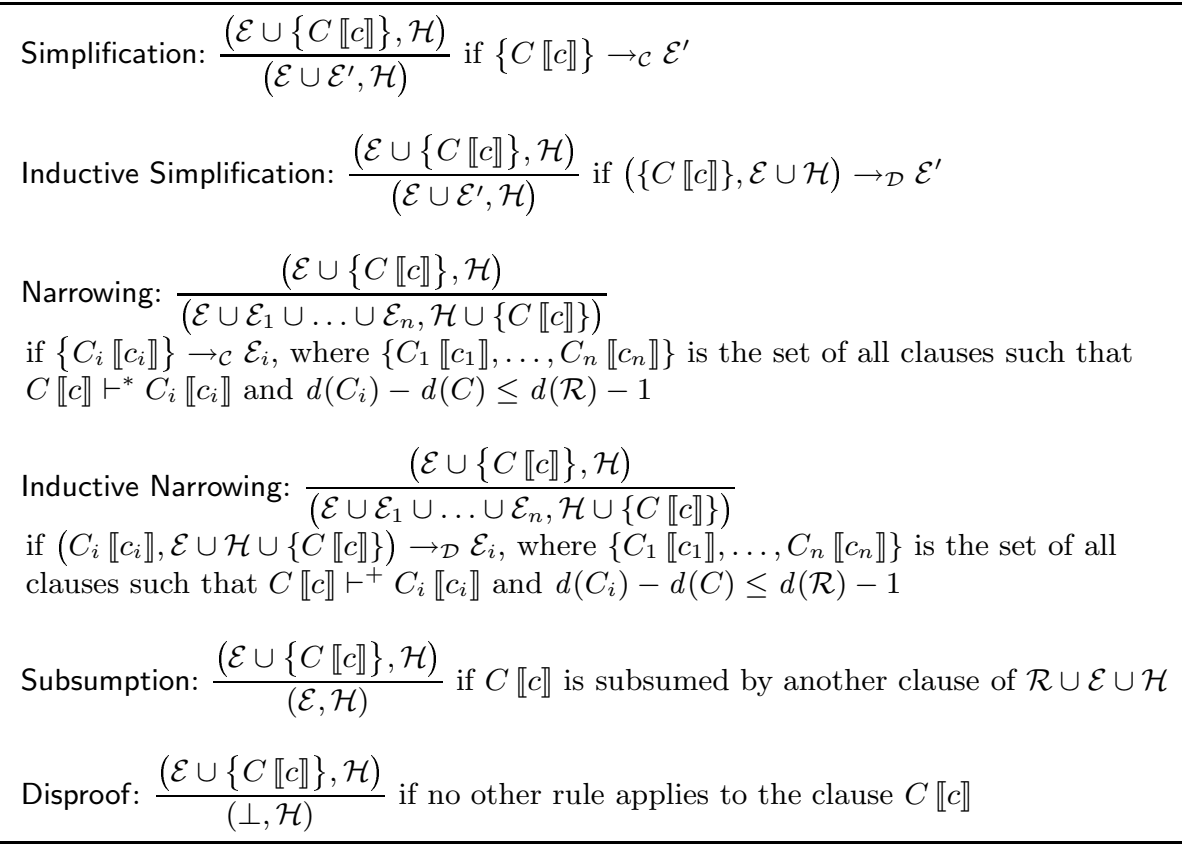

Fig. 3. Induction Inference Rules

\subsection{Induction Inference Rules}

The main inference system is displayed in Figure 3. Its rules apply to pairs $(\mathcal{E}, \mathcal{H})$ whose components are respectively the sets of current conjectures and of inductive hypotheses. Two inference rules below, Narrowing and Inductive Narrowing, use the grammar $\mathcal{G}_{\mathrm{NF}}\left(\mathcal{R}_{\mathcal{C}}\right)$ for instantiating variables. In order to be able to apply these inferences, we shall initiate the process by adding to the conjectures one membership constraint for each variable. 
Definition 3. Let $C \llbracket c \rrbracket$ be a constrained clause such that c contains no membership constraint. The decoration of $C \llbracket c \rrbracket$, denoted decorate $(C \llbracket c \rrbracket)$ is the set of clauses $C \llbracket c \wedge x_{1}:\left\llcorner u_{1}\right\lrcorner \wedge \ldots \wedge x_{n}:\left\llcorner u_{n} \rrbracket \rrbracket\right.$ where $\left\{x_{1}, \ldots, x_{n}\right\}=\operatorname{var}(C)$, and for all $i \in[1 . . n],{ }_{\llcorner} u_{i} \in Q_{\mathrm{NF}}\left(\mathcal{R}_{\mathcal{C}}\right)$ and $\operatorname{sort}\left(u_{i}\right)=\operatorname{sort}\left(x_{i}\right)$.

The definition of decorate is extended to set of constrained clauses as expected. A constrained clause $C \llbracket c \rrbracket$ is said decorated if $c=d \wedge x_{1}:\left\llcorner u_{1}\right\lrcorner \wedge \ldots \wedge x_{n}:\left\llcorner u_{n}\right\lrcorner$ where $\left\{x_{1}, \ldots, x_{n}\right\}=\operatorname{var}(C)$, and for all $i \in[1 . . n],\left\llcorner u_{i} \in Q_{\mathrm{NF}}\left(\mathcal{R}_{\mathcal{C}}\right), \operatorname{sort}\left(u_{i}\right)=\right.$ $\operatorname{sort}\left(x_{i}\right)$, and $d$ does not contain membership constraints.

Simplification, resp. Inductive Simplification, reduces conjectures according to the rules of Section 4.2, resp. 4.1, Inductive Narrowing generates new subgoals by application of the production rules of the constrained grammar $\mathcal{G}_{\mathrm{NF}}\left(\mathcal{R}_{\mathcal{C}}\right)$ until the obtained clause is deep enough to cover left-hand side of rules of $\mathcal{R}_{\mathcal{D}}$. Each obtained clause must be simplified by one the rules of Figure 1 (otherwise, if one instance cannot be simplified, then the rule Inductive Narrowing cannot be applied). For sake of efficiency, the application can be restricted to so called induction variables, as defined in [1] while preserving all the results of the next section. Narrowing is similar and uses the rules of Figure 2 for simplification. This rule permits to eliminate the ground reducible constructor terms in a clause by simplifying their instances, while deriving conjectures considered as new subgoals. The criteria on depth is the same for Inductive Narrowing and Narrowing and is a bit rough, for sake of clarity of the inference rules. However, in practice, it can be replaced by a tighter condition (with, e.g., a distinction between $\mathcal{R}_{\mathcal{C}}$ and $\mathcal{R}_{\mathcal{D}}$ ) while preserving the results of the next section. Subsumption deletes clauses redundant with axioms of $\mathcal{R}$, induction hypotheses of $\mathcal{H}$ and other conjectures not yet proved (in $\mathcal{E}$ ).

Example 3. Let us come back to the running example of sorted lists, with the constructor system $\mathcal{R}_{\mathcal{C}}$ containing $\left(\mathbb{C}_{0}\right)$ and $\left(\mathbb{C}_{1}\right)$ and the defined system $\mathcal{R}_{\mathcal{D}}$ containing the axioms $\left(\mathrm{m}_{0}^{\prime} \mid \mathrm{m}_{3}^{\prime}\right)$ given in introduction 1 together with the following axioms defining a variant $\in$ for the membership:

$$
\begin{array}{rlr}
x \in \emptyset & \rightarrow \text { false } & \left(\mathrm{m}_{0}\right) \\
x_{1} \in \text { ins }\left(x_{2}, y\right) & \rightarrow \text { true } \llbracket x_{1} \approx x_{2} \rrbracket & \left(\mathrm{m}_{1}\right) \\
x_{1} \in \text { ins }\left(x_{2}, y\right) & \rightarrow x_{1} \in y \llbracket x_{1} \not x_{2} \rrbracket & \left(\mathrm{m}_{2}\right)
\end{array}
$$

We show, using our procedure, that the conjecture $x \Subset y=x \in y$ is an inductive theorem of $\mathcal{R}$, i.e. that the two variants $\Subset$ and $\in$ of membership are equivalent. The normal-form grammar $\mathcal{G}_{\mathrm{NF}}\left(\mathcal{R}_{\mathcal{C}}\right)$ is described in example 2, The decoration of the conjecture with its non-terminal gives the two clauses: $x \Subset y=x \in$ $y \llbracket x:\left\llcorner x_{\lrcorner}^{\mathrm{Nat}}, y:\left\llcorner x_{\lrcorner}^{\mathrm{Set}} \rrbracket\right.\right.$ and $x \Subset y=x \in y \llbracket x:\left\llcorner x_{\lrcorner}^{\mathrm{Nat}}, y: \operatorname{ins}\left(x_{1}, y_{1}\right)\right\lrcorner \rrbracket$.

The application of the production rules of $\mathcal{G}_{\mathrm{NF}}\left(\mathcal{R}_{\mathcal{C}}\right)$ to the first of these clauses (in Narrowing) gives: $x \Subset \emptyset=x \in \emptyset$ which is reduced, using $\left(\mathrm{m}_{0}^{\prime}\right)$ and $\left(\mathrm{m}_{0}\right)$, to the tautology false $=$ false. For the second clause, applying $\mathcal{G}_{\mathrm{NF}}\left(\mathcal{R}_{\mathcal{C}}\right)$ returns:

$$
x \Subset \operatorname{ins}\left(x_{1}, \emptyset\right)=x \in \operatorname{ins}\left(x_{1}, \emptyset\right) \llbracket x, x_{1}:\left\llcorner x_{\lrcorner}^{\mathrm{Nat}} \rrbracket\right.
$$

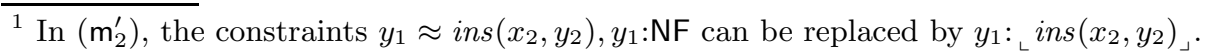




$$
\begin{aligned}
x \Subset \operatorname{ins}\left(x_{1}, \operatorname{ins}\left(x_{2}, \emptyset\right)\right) & =x \in \operatorname{ins}\left(x_{1}, \operatorname{ins}\left(x_{2}, \emptyset\right)\right) \llbracket x, x_{1}, x_{2}:\left\llcorner x_{\lrcorner}^{\mathrm{Nat}}, x_{1} \prec x_{2} \rrbracket\right. \\
x \Subset \operatorname{ins}\left(x_{1}, \text { ins }\left(x_{2}, y_{2}\right)\right) & =x \in \operatorname{ins}\left(x_{1}, \text { ins }\left(x_{2}, y_{2}\right)\right) \\
& \llbracket x, x_{1}, x_{2}:\left\llcorner x_{\lrcorner}^{\mathrm{Nat}}, y_{2}:\left\llcorner\operatorname{ins}\left(x_{3}, y_{3}\right), x_{1} \prec x_{2}, x_{2} \prec x_{3} \rrbracket\right.\right.
\end{aligned}
$$

The subgoals (11) and (2) can be simplified by Rewrite Splitting with $\left(\mathrm{m}_{1}^{\prime}, \mathrm{m}_{2}^{\prime}\right.$ and $\left(\mathrm{m}_{3}^{\prime}\right)$ into clauses reduced into tautologies (see 3 for details).

The subgoal (3) is implified by Rewrite Splitting with $\left(\mathrm{m}_{1}^{\prime} \sqrt{\mathrm{m}_{3}^{\prime}}\right)$ into 3 clauses. Let us consider the third one, obtained with $\left(\overrightarrow{\mathrm{m}_{3}^{\prime}}\right): x \Subset$ ins $\left(x_{2}, y_{2}\right)=x \in$ $\operatorname{ins}\left(x_{1}, \operatorname{ins}\left(x_{2}, y_{2}\right)\right) \llbracket x, x_{1}, x_{2}, x_{3}:\left\llcorner x_{\lrcorner}^{\mathrm{Nat}}, y_{2}:\right.$ ins $\left.\left(x_{3}, y_{3}\right)\right\lrcorner, x_{1} \prec x_{2}, x_{2} \prec x_{3}, x_{1} \prec x \rrbracket$. It is simplified by Inductive Rewriting with $\left(\mathrm{m}_{2}\right)$ into: $x \Subset \operatorname{ins}\left(x_{2}, y_{2}\right)=x \in \operatorname{ins}\left(x_{2}, y_{2}\right) \llbracket x, x_{2}, x_{3}:\left\llcorner x_{\lrcorner}^{\mathrm{Nat}}, y_{2}:\right.$ ins $\left.\left(x_{3}, y_{3}\right)\right\lrcorner, x_{2} \prec x_{3} \rrbracket$.

At this point, we are allowed to use the conjecture $x \stackrel{\complement}{\Subset} y=x \in y$ as an induction hypothesis with Inductive Rewriting, it returns the tautology:

$$
x \Subset \operatorname{ins}\left(x_{2}, y_{2}\right)=x \Subset \operatorname{ins}\left(x_{2}, y_{2}\right) \llbracket x, x_{2}, x_{3}:\left\llcorner x_{\lrcorner}^{\mathrm{Nat}}, y_{2}:\left\llcorner\operatorname{ins}\left(x_{3}, y_{3}\right)\right\lrcorner, x_{2} \prec x_{3} \rrbracket\right.
$$

The ommited details in the proof of the conjecture can be found in [3]. Note that this proof does not require the manual addition of lemma.

\subsection{Soundness and Completeness}

We show now that our inference system is sound and refutationally complete. The proof of soundness is not straightforward. The main difficulty is to make sure that the exhaustve application of the rules preserve a counterexample when one exists. We will show more precisely that a minimal counterexample is preserved along a fair derivation.

A derivation is a sequence of inference steps generated by a pair of the form $\left(\mathcal{E}_{0}, \emptyset\right)$, using the inference rules in $\mathcal{I}$, written $\left(\mathcal{E}_{0}, \emptyset\right) \vdash_{\mathcal{I}}\left(\mathcal{E}_{1}, \mathcal{H}_{1}\right) \vdash_{\mathcal{I}} \ldots$ It is called fair if the set of persistent constrained clauses $\left(\cup_{i} \cap_{j \geq i} \mathcal{E}_{j}\right)$ is empty or equal to $\{\perp\}$. The derivation is said to be a disproof in the latter case, and a success in the former.

Finite success is obtained when the set of conjectures to be proved is exhausted. Infinite success is obtained when the procedure diverges, assuming fairness. When it happens, the clue is to guess some lemmas which are used to subsume or simplify the generated infinite family of subgoals, therefore stopping the divergence. This is possible in principle with our approach, since lemmas can be specified in the same way as axioms are.

Theorem 1 (Soundness of successful derivations). Assume that $\mathcal{R}_{\mathcal{C}}$ is terminating and that $\mathcal{R}$ is sufficiently complete. Let $\mathcal{D}_{0}$ be a set of unconstrained clauses and let $\mathcal{E}_{0}=$ decorate $\left(\mathcal{D}_{0}\right)$. If there exists a successful derivation $\left(\mathcal{E}_{0}, \emptyset\right) \vdash_{\mathcal{I}}\left(\mathcal{E}_{1}, \mathcal{H}_{1}\right) \vdash_{\mathcal{I}} \cdots$ then $\mathcal{R} \models \models_{\text {Ind }} \mathcal{D}_{0}$.

Proof. (sketch, see [3] for a complete proof). The proof uses the fact that, under the hypotheses of Theorem $1 \mathcal{R} \models \models_{\text {Ind }} \mathcal{E}_{0}$ implies $\mathcal{R} \models_{\text {Ind }} \mathcal{D}_{0}$.

Intuitively, the reason is that in order to show that $\mathcal{R} \models_{\mathcal{I}_{n d}} \mathcal{D}_{0}$, it is sufficient to show that $\mathcal{R} \models \mathcal{D}_{0} \sigma$ for all substitutions $\sigma$ whose images contain only ground 
constructor terms in normal form. Every ground $\sigma$ can indeed be normalized into a substitution of this form because $\mathcal{R}_{\mathcal{C}}$ is terminating and $\mathcal{R}$ sufficiently complete. By definition of the decoration, the membership constraints and by construction of $\mathcal{G}_{\mathrm{NF}}\left(\mathcal{R}_{\mathcal{C}}\right)$, this sufficient condition is a consequence of $\mathcal{R} \models_{\mathcal{I n d}} \mathcal{E}_{0}$.

We then show that $\mathcal{R} \models_{\text {Ind }} \mathcal{E}_{0}$ by minimal counter-example. Assume that $\mathcal{R} \nvdash_{\mathcal{I} n d} \mathcal{E}_{0}$ and let $D_{0}$ be a clause, minimal wrt $\gg$, in the set:

$\left\{D \sigma \mid D \llbracket d \rrbracket \in \cup_{i} \mathcal{E}_{i}, \sigma \in \operatorname{sol}(d)\right.$ is constructor and irreducible and $\left.\mathcal{R} \not \models D \sigma\right\}$.

Let $C \llbracket c \rrbracket$ be a clause of $\cup_{i} \mathcal{E}_{i}$ minimal by subsumption ordering and $\theta \in \operatorname{sol}(c)$, irreducible and constructor ground substitution, be such that $C \theta=D_{0}$. We show in [3] that whatever inference, other than Disproof, is applied to $C \llbracket c \rrbracket$, a contradiction is obtained, hence that the above derivation is not successful.

Since there are only two kinds of fair derivations, we obtain as a corollary:

Corollary 1 (Refutational completeness). Assume that $\mathcal{R}_{\mathcal{C}}$ is terminating and that $\mathcal{R}$ is sufficiently complete. Let $\mathcal{D}_{0}$ be a set of unconstrained clauses and let $\mathcal{E}_{0}=\operatorname{decorate}\left(\mathcal{D}_{0}\right)$. If $\mathcal{R} \forall_{\mathcal{I n d}} \mathcal{E}_{0}$, then all fair derivations starting from $\left(\mathcal{E}_{0}, \emptyset\right)$ end up with $(\perp, \mathcal{H})$.

When we assume that all the variables in goals are decorated (restricting the domain for this variables to ground constructor irreducible terms), the above hypotheses that $\mathcal{R}_{\mathcal{C}}$ is terminating and $\mathcal{R}$ is sufficiently complete can be dropped.

Theorem 2 (Soundness of successful derivations). Let $\mathcal{E}_{0}$ be a set of decorated constrained clauses. If there exists a successful derivation $\left(\mathcal{E}_{0}, \emptyset\right) \vdash_{\mathcal{I}}$ $\left(\mathcal{E}_{1}, \mathcal{H}_{1}\right) \vdash_{\mathcal{I}} \cdots$ then $\mathcal{R} \models_{\text {Ind }} \mathcal{E}_{0}$.

Proof. (sketch). We use the second part of the proof of Theorem 1 (which does not use the sufficient completeness of $\mathcal{R}$ and termination of $\mathcal{R}_{\mathcal{C}}$ ). With the hypothesis that the clauses of $\mathcal{E}_{0}$ are decorated, the fact given at the beginning of this proof is indeed no more needed $\left(\mathcal{D}_{0}=\mathcal{E}_{0}\right)$. The restriction to substitutions into ground constructor normal forms in order to show that $\mathcal{R} \nvdash_{\mathcal{I} n d} \mathcal{E}_{0}$ is made explicit by the membership constraints in the decoration.

Corollary 2 (Refutational completeness). Let $\mathcal{E}_{0}$ be a set of decorated constrained clauses. If $\mathcal{R} \nvdash_{\mathcal{I} n d} \mathcal{E}_{0}$, then all fair derivations starting from $\left(\mathcal{E}_{0}, \emptyset\right)$ end up with $(\perp, \mathcal{H})$.

We shall see in Section 5 some example of applications of Theorem 2 and Corollary 2 to specifications which are not sufficiently complete.

Theorem 3 (Soundness of disproof). Assume that $\mathcal{R}$ is strongly complete and ground confluent. If a derivation starting from $\left(\mathcal{E}_{0}, \emptyset\right)$ returns the pair $(\perp, \mathcal{H})$, then $\mathcal{R} \forall_{\text {Ind }} \mathcal{E}_{0}$. 


\subsection{Handling Non-terminating Constructor Systems}

Our procedure applies rules of $\mathcal{R}_{\mathcal{C}}$ and $\mathcal{R}_{\mathcal{D}}$ only when they reduce the terms wrt the given simplification ordering $>$. This is ensured when the rewrite relation induced by $\mathcal{R}_{\mathcal{C}}$ and $\mathcal{R}_{\mathcal{D}}$ is compatible with $>$, and hence that $\mathcal{R}_{\mathcal{C}}$ and $\mathcal{R}_{\mathcal{D}}$ are terminating (separately). Note that this is in contrast with other procedures like [16] where the termination of the whole system $\mathcal{R}$ is required.

If $\mathcal{R}_{\mathcal{C}}$ is non-terminating then one can apply a constrained completion technique [16 in order to generate an equivalent orientable theory (with ordering constraints). The theory obtained (if the completion succeeds) can then be handled by our approach.

Example 4. Consider this non-terminating system for sets: $\{\operatorname{ins}(x, \operatorname{ins}(x, y))=$ ins $(x, y)$, ins $\left(x\right.$, ins $\left.\left(x^{\prime}, y\right)\right)=\operatorname{ins}\left(x^{\prime}\right.$, ins $\left.\left.(x, y)\right)\right\}$. Applying the completion procedure we obtain the constrained rules $\left(\mathbb{C}_{0}\right)$ and $\left(\boldsymbol{C}_{1}\right)$.

\subsection{Decision Procedures for Conditions in Inferences}

Constrained tree grammars are involved in the inferences Narrowing and Inductive Narrowing in order to generate subgoals from goals, by instantiation using the productions rules. They are also the key for the decision procedures applied in order to check the conditions of constraint unsatisfiability (in rules for rewriting and Inductive Deletion, Deletion, Subsumption), ground irreducibility and validity of ground irreducible constructor clauses (in the rules Validity, hence Simplification, and Disproof). These conditions are decided by reduction into the decision problem of emptiness (of $L\left(\mathcal{G},\left\llcorner u_{\lrcorner}\right)\right.$) for constrained tree grammars build from $\mathcal{G}_{\mathrm{NF}}\left(\mathcal{R}_{\mathcal{C}}\right)$. The decision rely on similar decision results for constrained tree automata, some cases are summarized in $[8]$. The reductions are detailed in [3].

\section{$5 \quad$ Handling Partial Specifications}

The example of sorted lists (Example 3) can be treated with our procedure because it is based on a sufficiently complete and ground confluent conditional constrained TRS $\mathcal{R}$ whose constructor part $\mathcal{R}_{\mathcal{C}}$ is terminating. Indeed, under these hypotheses, Theorem 1 ensures the soundness of our procedure for proving inductive conjectures on this specification, and Corollary 1 and Theorem 3 ensure respectively refutational completeness and soundness of disproof.

For sound proofs of inductive theorems wrt specifications which are not sufficiently complete, we can rely on Theorem 2 and Corollary 2 which do not require sufficient completeness of the specification but instead suppose that the conjecture is decorated, i.e. that each of its variables is constrained to belong to a language associated to a non-terminal of the normal-form (constrained) grammar. In this section, we propose two applications of this principle of decoration of conjectures to the treatment of partial specifications.

Partially Defined Functions. An inductive proof of a decorated conjecture $C$ in $\mathcal{R}$ remains valid in an extension of $\mathcal{R}$ (possibly not complete). 
Theorem 4. Assume that $\mathcal{R}$ is sufficiently complete and let $\mathcal{R}^{\prime}$ be an consistent extension of $\mathcal{R}$ where $\mathcal{R}_{\mathcal{C}}{ }^{\prime}=\mathcal{R}_{\mathcal{C}}$ and $\mathcal{R}_{\mathcal{D}}{ }^{\prime}=\mathcal{R}_{\mathcal{D}} \cup \mathcal{R}_{\mathcal{D}}{ }^{\prime \prime}\left(\mathcal{R}_{\mathcal{D}}{ }^{\prime \prime}\right.$ defines additional partial defined functions). Let $\mathcal{E}_{0}$ be a set of decorated constrained clauses. Every derivation $\left(\mathcal{E}_{0}, \emptyset\right) \vdash_{\mathcal{I}} \cdots$ successful wrt $\mathcal{R}$ is also a successful derivation wrt $\mathcal{R}^{\prime}$.

In [3, we use Theorem 4 for the proof of conjectures on an extension of the specification of Example 3 with the incomplete definition of a function min.

Partial Constructors. The restriction to decorated conjectures also permits to deal with partial constructor functions. In this case, we are generally interested in proving conjectures only for constructor terms in the definition domain of the defined function (well-formed terms).

In [3, we present an example of automatic proof where $\mathcal{R}_{\mathcal{C}}$ is such that the set of well-formed terms is the set of constructor $\mathcal{R}_{\mathcal{C}}$-normal forms. Hence, decorating the conjecture with grammar's non-terminals, as in Theorem 2 amounts in this case at restricting the variables to be instantiated by well-formed terms.

The example is a specification of powerlists (lists of $2^{n}$ integers stored in the leaves of a complete binary tree) also treated in [15]. A particularity of this example is that $\mathcal{R}_{\mathcal{C}}$ contains constraints of the form $t \sim t^{\prime}$ meaning that $t$ and $t^{\prime}$ are well-formed lists of the same length (i.e. balanced trees of the same depth). Such constraints are added to $\mathcal{G}_{\mathrm{NF}}\left(\mathcal{R}_{\mathcal{C}}\right)$ and we show that emptiness is decidable for these grammars by reduction to the same problem for visibly tree automata with one memory [11].

\section{Conclusion}

We have proposed a procedure for automated inductive theorem proving in specification made of conditional and constrained rewrite rules. Constraints in rules can serve to transform non terminating specifications into terminating ones (ordering constraints), define ad-hoc evaluation strategies (normal form constraints), or for the analysis of trace properties of infinite state systems like security protocols (constraints of membership in a regular tree language representing faulty traces [2]). The expressiveness and efficiency of the procedure are obtained by the use of constrained tree grammars as a finite representation of the initial model of specifications. They are used both as induction schema and for decision procedures.

The procedure presented in this paper is currently under implementation, on the top of the theorem prover SPASS, whose main loop is used for TA decision procedure by saturation, following the approach of e.g. [14.

\section{Acknowledgments}

We wish to thank Michael Rusinowitch, Hubert Comon-Lundh, Laurent Fribourg and Deepak Kapur for the fruitful discussions that we had together regarding this work. We are also grateful to Jared Davis and Sorin Stratulat for having processed the example on sorted lists with respectively ACL2 and SPIKE. 


\section{References}

1. Bouhoula, A.: Automated theorem proving by test set induction. Journal of Symbolic Computation 23(1), 47-77 (1997)

2. Bouhoula, A., Jacquemard, F.: Verifying regular trace properties of security protocols with explicit destructors and implicit induction. In: Proc. of the workshop FCS-ARSPA, pp. 27-44 (2007)

3. Bouhoula, A., Jacquemard, F.: Automated induction with constrained tree automata. Research Report LSV-08-07, http://www.lsv.ens-cachan.fr/Publis

4. Bouhoula, A., Jouannaud, J.-P.: Automata-driven automated induction. Information and Computation 169(1), 1-22 (2001)

5. Bouhoula, A., Jouannaud, J.-P., Meseguer, J.: Specification and proof in membership equational logic. Theoretical Computer Science 236(1-2), 35-132 (2000)

6. Bouhoula, A., Rusinowitch, M.: Implicit induction in conditional theories. Journal of Automated Reasoning 14(2), 189-235 (1995)

7. Comon, H.: Unification et disunification. Théories et applications. PhD thesis, Institut Polytechnique de Grenoble (France) (1988)

8. H. Comon, M. Dauchet, R. Gilleron, F. Jacquemard, C. Löding, D. Lugiez, S. Tison, and M. Tommasi. Tree automata techniques and applications (2007), http://www.grappa.univ-lille3.fr/tata

9. Comon, H., Jacquemard, F.: Ground reducibility is exptime-complete. Information and Computation 187(1), 123-153 (2003)

10. Comon-Lundh, H.: Handbook of Automated Reasoning, ch. 14. Elsevier, Amsterdam (2001)

11. Comon-Lundh, H., Jacquemard, F., Perrin, N.: Tree automata with memory, visibility and structural constraints. In: Seidl, H. (ed.) FOSSACS 2007. LNCS, vol. 4423, pp. 168-182. Springer, Heidelberg (2007)

12. Davis, J.: Finite set theory based on fully ordered lists. In: In 5th International Workshop on the ACL2 Theorem Prover and Its Applications (ACL2, 2004) Sets Library Website (2004), http://www.cs.utexas.edu/users/jared/osets/Web

13. Dershowitz, N., Jouannaud, J.-P.: Rewrite systems. In: Handbook of Theoretical Computer Science, Volume B: Formal Models and Sematics, pp. 243-320. MIT Press, Cambridge (1990)

14. Jacquemard, F., Rusinowitch, M., Vigneron, L.: Tree automata with equality constraints modulo equational theories. Journal of Logic and Algebraic Programming (to appear, 2008)

15. Kapur, D.: Constructors can be partial too. In: Essays in Honor of Larry Wos, MIT Press, Cambridge (1997)

16. Kirchner, C., Kirchner, H., Rusinowitch, M.: Deduction with symbolic constraints. Revue d'Intelligence Artificielle 4(3), 9-52 (1990); Special issue on Automatic Deduction

17. Stratulat, S.: A general framework to build contextual cover set induction provers. Journal of Symbolic Computation 32(4), 403-445 (2001)

18. Zhang, H.: Implementing contextual rewriting. In: Proc. 3rd Int. Workshop on Conditional Term Rewriting Systems (1992) 